\title{
ASSESSMENT OF CANCER RELATED FATIGUE
}

\author{
R. Strebkova*, M. Petkova, M. Minev
}

\author{
Department of Medical Psychology and Foreign Languages, Medical Faculty, Trakia University, \\ Stara Zagora, Bulgaria
}

\begin{abstract}
Cancer-related fatigue is one of the most common and significant symptoms experienced by cancer patients. The cancer-related fatigue can occur at any stage of the disease or its treatment and has an impact on patient's quality of life, regarding physical functioning and the ability to perform activities of daily living. In addition, it is associated with psychological distress. There is no agreement between various authors about the definition and measurement of cancer-related fatigue. There is a range of scales which measure cancer-related fatigue based on unidimensional or multi-dimensional perspective. This review aims to identify available tools applied in practice for the measurement and evaluation of cancer-related fatigue and discusses important conceptual and psychometric characteristics of these instruments.
\end{abstract}

Key words: Cancer, Fatigue, Assessment, Anxiety, Depression

\section{INTRODUCTION}

Cancer-related fatigue has been defined as „Sustainable subjective feeling of tiredness, related to the oncological disease or its treatment, which interferes with normal functioning" (1). Fatigue is a widespread symptom in cancer patients and cancer survivors, which can occur in any stage of disease, during the treatment (2), in advanced stage of disease (3), as well as in cancer survivors (4) and to last for a long period after completion of treatment. According to the upto-date definition by National Comprehensive Cancer Network (5), cancer-related fatigue is a limitation of physical and mental capacity, which occurs in relation to the medical treatment, has a significant impact on patient's activity and cannot be reduced despite adequate rest and relaxation. Therefore, the term "fatigue" as tiredness is not sufficient to cover a range of various manifestations of the problem. $(6,7)$ define cancer-related fatigue as multi-dimensional construct with typical symptoms; physical (physical exhaustion, fatigue), psychic (depressed mood, sadness, irritability, hopelessness) and cognitive (impaired concentration and memory).

\footnotetext{
*Correspondence to: Raisa Strebkova, Trakia University, Medical Faculty, Department of "Medical psychology and foreign languages", Armeyska Street N11, Stara Zagora. E-mail: rajal@abv.bg
}

Spreading of cancer-related fatigue described in medical literature varies widely $14-99 \%$ (5, 8). At the stage of diagnosis, up to $40 \%$ of patients complain of fatigue, this percentage grows up to $90 \%$ in the course of treatment (9). Almost all patients experience fatigue during the treatment of an oncological disease - with $90 \%$ of patients treated with radiotherapy, and with $80 \%$ of patients treated with chemotherapy. The frequency of fatigue can vary widely, also in dependence on what measurement tool has been used (10). Recognition of the importance of fatigue for patients' psychosocial and cognitive functioning, its impact on the affected people's quality of life, has enhanced the development of a wide range of instruments for assessment and diagnosis of this symptom (11).

Over 20 different tools have been applied in practice in order to assess one or more dimensions of cancer-related fatigue. Unidimensional tools are normally focused on the identification of the occurrence and severity of fatigue, while multi-dimensional ones examine its impact on physical, social, emotional, and cognitive functioning. Development of guidelines for cancer-related fatigue by National Comprehensive Cancer Network (NCCN) (12), the initial formulation, preliminary tests for diagnosis are the state-ofart achievements in assessment and management of this symptom. 
The aim of this study is to review the available scales for measurement of cancer-related fatigue and discuss their clinical and psychometric characteristics. This review would be a useful step towards the achievement of overall assessment of the fatigue in clinical practice, as well as its recognition and management $(13,14)$.

\section{QUESTIONNAIRES FOR ASSESSMENT OF FATIGUE, USED ALONE OR IN COMBINATION WITH OTHER TOOLS.} 1. VAFS (Visual Analog Fatigue Scale) (15). VAFS is used for assesses fatigue severity. Present 10-cm line with 2 end points "I do not feel tired at all" and "I feel totally exhausted". Measurements are performed four times on each test day, at 7.00, 12.00, 17.00 and 21 hours. The scale is able to detect specific fatigue profiles over a daily period in healthy persons and in cancer patients.

\section{HADS (Hospital Anxiety and Depression Scale)(16).}

Selfassessment questionnaire to determine the presence of intense anxiety and depressive conditions. It contains 2 subscales: anxiety and depression, and 14 questions that assess independently nonsomatic symptoms of anxiety (HADS-A) and depression (HADS-D). The possible scores for depression and anxiety range from 0 to 21 with normal scores ranging from 0-7 and scores of 8-10 indicating borderline depression or anxiety. The internal consistency is good for both subscales, respectively, anxiety and depression; $(\alpha=$ $0.785 ; \alpha=0.841)$.

\section{UNIDIMENSIONAL QUESTIONNAIRES USED FOR THE ASSESSMENT OF FATIGUE.}

\section{BFI (Brief Fatigue Inventory) (17)}

The Brief Fatigue Inventory is used to rapidly assess the severity and impact of cancer-related fatigue on daily functioning over the past 24 hours. There are 9 items in the scale. Three items ask patients to rate the severity of their fatigue at its "worst," "usual," and "now" during normal waking hours, with 0 being "no fatigue" and 10 being "fatigue as bad as you can imagine." Six items assess the amount that fatigue has interfered with different aspects of the patient's life during the past 24 hours. The interference items include general activity, mood, walking ability, normal work (includes both work outside the home and housework), relations with other people, and enjoyment of life. The interference items are measured on a $0-10$ scale, with 0 being "does not interfere" and 10 being "completely interferes." Internal consistency $(\alpha=0.96)$
2. EORTC QLQ-C30 (European Organization for Research and Treatment of Cancer Quality of Life Questionnaire - C - 30) v. 3.0 (18).

The EORTC
$\begin{aligned} & \text { QLQ-C30 (V 3.0) is an } \\ & \text { internationally } \\ & \text { validated thirty-item }\end{aligned}$
specifically designed for multidimensional measurement of QOL in cancer patients. It includes five functioning scales (physical, role, emotional, cognitive and social functioning), three symptom scales (fatigue,pain nausea and vomiting), and six single items (dyspnea, sleep disturbance, loss of appetite, constipation, diarrhoea and financial difficulties). It also includes two questions on patient overall QOL and overall physical condition, allowing a global QOL score to be obtained. The total score is calculated from the scales and single items and ranges from 0 to 100 . A high total score for functioning scales and global QOL scale indicates a high level of functioning or QOL. Additionally global health and overall quality of life is measured. The three-item fatigue subscale has been independently validated as a separate fatigue measure. There have also been two largescale studies (>2000 patients in each) independently assessing its use $(19 ; 20)$, so there are extensive data in a variety of settings. However, it has been noted to have a ceiling effect in advanced cancer patients (19) and is not recommended as a single measure in this group. Internal consistency for most scales $(\alpha=0.80)$.

\section{MULTIDIMENSIONAL QUESTIONNAIRES USED FOR THE} ASSESSMENT OF FATIGUE.

\section{MFI-20 - Multidimensional Fatigue \\ Inventory (21).}

The Multidimensional Fatigue Inventory has been validated in patients with cancer and focuses on the subjective experience of fatigue. This scale evaluates the general, mental, and physical dimensions of fatigue, as well as levels of motivation and activity. The 20-item version of the MFI has been used to assess fatigue in patients with a variety of cancers who are receiving chemotherapy or radiotherapy (22). Using this scale, higher levels of fatigue have been detected during and immediately after treatment. One study confirmed higher levels of fatigue across all five dimensions measured (general,physical, activity, motivation, and mental) in patients (23). The MFI has also been used to demonstrate the persistence of multiple dimensions of fatigue after completion of initial treatment in a range of cancers $(24,25)$ receiving anticancer treatment compared with 
healthy individuals. The instrument has good internal consistency $(\alpha=0.84)$.

\section{FQ (Chalder Fatigue Scale/Fatigue Questionnaire) (26).}

The Chalder Fatigue Scale is an 11-item scale that was originally validated in a general practice setting. However, its main use has been in the investigation of chronic fatigue syndrome. It is brief and easy to administer while still covering two aspects of fatigue (mental and physical). It has been used in population studies and so has normative data available for comparison with cancer patients (27; 28). Internal consistency ( $\alpha=0.88-0.90)$.

\section{FSI - Fatigue Symptom Inventory (29)}

The Fatigue Symptom Inventory (FSI) is a 13item scale and $0-10$ numeric rating scale that was originally validated in a breast cancer population. This scale measures the severity, frequency, diurnal variation, and interference with quality of life resulting from fatigue over the previous 7 days. It has reasonable psychometric properties but there is a question over its test-retest reliability. The FSI has mainly been used in studies examining the effect of chemotherapy for breast cancer (30; 31). These studies have shown that the majority of patients with breast cancer who receive chemotherapy are likely to experience some degree of CRF. More importantly, these studies have shown that the CRF these patients reported was more severe than normal fatigue reported by healthy controls. In follow-up studies using the FSI, patients with breast cancer reported significantly more fatigue for longer periods-months and even years following completion of treatment $(32,29)$.

\section{PFS - Piper Fatigue Scale (33).}

The revised Piper Fatigue Scale (PFS)was primarily validated in patients with breast cancer and, as such, might have limited generalizability to patients with other cancer diagnoses. It is used for assess fatigue severity. Assesses 4 dimensions of fatigue - behavioral, affective, sensory, and cognitive. Contains 22 items and 0-10 numerical rating scale. Longitudinal studies using the PFS as a measure of fatigue have demonstrated persistent and elevated levels of fatigue across multiple domains in patients with various cancer diagnoses compared with controls (34; 35). There are limited data on the psychometric properties of the revised scale for use in cancer patients although data are available in other populations. Internal consistency $(\alpha=0.97)$. Total score $(\alpha=0.96)$, and the different scales, behavioral, affective, sensory and cognitive dimension respectively $(\alpha=0.896 ; \alpha=0.96 ; \alpha=0.96$ and $\alpha=0.91)$ (36).

\section{SCFS - Schwartz Cancer Fatigue Scale (37).}

The Schwartz Cancer Fatigue Scale is a 28item scale and 1-5 rating scale with 1, "not at all," to 5 "extremely", that was validated in a mixed cancer population undergoing treatment. Assesses 4 dimensions of fatigue - physical, emotional, cognitive, and temporal "In the prior 2 to 3 days". The Schwartz Fatigue Scale has been used successfully in a number of interventional trials to assess the impact of exercise on the experience of fatigue in women with breast cancer $(38 ; 39)$. Construct validity was proved by differences of established fatigue among patients undergoing treatment at the moment, and those who have completed treatment and results of visual analogue scale for fatigue. Internal consistency $(\alpha=0.96)$.

\section{FAQ-Fatigue Assessment Questionnaire} (40).

FAQ characterizes fatigue in terms of physical, affective, and cognitive aspects. Contains 20 items, (11 items of physical,5 of affective, and 3 of cognitive fatigue) plus a single item on sleep disturbance, each assessed on a 4-point scale. The FAQ has been developed based on thorough interviews and tested on a large mixed sample of cancer patients. Internal consistency of the questionnaire was supported by the Alpha Coefficient 0.90 (Cronbach). A difference was found in physical tiredness for patients with different types of cancer $(\mathrm{p}=$ 0.008 ) but this was not the case for affective and cognitive tiredness. More fatigue feelings were experienced by cancer patients with advanced disease than by patients in remission and this was true for physical fatigue ( $\mathrm{p}=$ $0.0001)$, affective fatigue ( $\mathrm{p}=0.01)$, cognitive fatigue $(\mathrm{p}=0.02)$ as well as for fatigue intensity $(\mathrm{p}=0.0001)$ and distress $(\mathrm{p}=$ 0.0001). This group of patients also experienced significantly higher levels of depression and anxiety $(\mathrm{p}=0.0001)$.

\section{CFS-Cancer Fatigue Scale (41).}

The CFS is a 15-item scale composed of 3 subscales (physical, affective, and cognitive subscales) and 1-5 numeric rating scale with 1 , "no," to 5, "very much." Three hundred seven cancer patients participated in the validation phase. The CFS had good stability (average test-retest reliability $\mathrm{r}=0.69, \mathrm{P}<0.001$ ) and good internal consistency (Cronbach's alpha coefficient for all 15 items $=0.88$ ). 


\section{DISCUSSION}

Cancer-related fatigue is a highly prevalent and debilitating symptom experienced by most cancer patients during, and often for considerable periods after treatment. The recognition of the importance of CRF to patients' psychosocial and cognitive functioning, as well as to their quality of life, has driven the development of a wide range of assessment tools for screening and diagnosis of Cancer related fatigue. The examination carried out showed the range and quantity of available and validated tools for specific measurement and reliable assessment of cancer-related fatigue. Many of the tools differ in terms of their psychometric characteristics. The unidimensional ones examine the availability or lack of fatigue. A small number of them focus on the severity of tumour-related fatigue and its relation to various aspects of patients' lives. The multi-dimensional tools examine the influence of fatigue on physical, emotional, cognitive and social functioning of affected patients, but a few of them cover all the three - physical, emotional and cognitive components of fatigue $(37 ; 41 ; \underline{42})$. The full assessment of fatigue and psychic functioning in cancer patients requires combination between multidimensional cancer-related fatigue questionnaires and specific psychological scales (anxiety and depression scales - HADS) (43).

\section{CONCLUSION}

1. The presence of multiple scales to assess cancer-related fatigue, which can be divided into two main categories.

2. Unidimensional measures are often singlequestion scales that generally focus on identifying the occurrence and severity of cancer-related fatigue.

3. Multidimensional scales measures physical, behavioral, affective, sensory, and cognitive aspects of cancer related fatigue.

4. The full assessment of fatigue and psychological functioning in cancer patients requires combination between multidimensional cancer related fatigue questionnaires and specific psychological scales (anxiety and depression scales).

5. Adequate monitoring of cancer-related fatigue can contribute to the development more appropriate treatment strategies, palliative or curative, which in turn may enhance understanding of this syndrome.

\section{REFERENCES}

1. Mock, V. Atkinson, A. Barsevick, A. Cella, D. Cimprich, B. Cleeland, C., et al., NCCN Practice Guidelines for Cancer-Related
Fatigue. Oncology (Williston Park), 14:151-61, 2000

2. Stone, P. Richards, M. A'Hern, R. Hardy, J., Fatigue in patients with cancers of the breast or prostate undergoing radical radiotherapy. Journal of Pain and Symptom Management, 22:1007-15.[PubMed: 11738163] 2001

3. Stone, P. Hardy, J. Broadley, K. Tookman, AJ. Kurowska, A. Hern, RA., Fatigue in advanced cancer: A prospective controlled cross-sectional study. British Journal of Cancer,79:1479-86. [PubMed:10188894] 1999

4. Bower, JE. Ganz, PA. Desmond, KA. et al., Fatigue in long-term breast carcinoma survivors. Cancer, 106(4):751-758, 2006

5. NCCN (National Comprehensive Cancer Network). Clinical practice guidelines in oncology: Cancer related fatigue, 2011

6. Kuhnt, S. Brähler, E., Tumorassoziierte Fatigue. Psychother Psych Med, 60:402411, 2010

7. Petkova, M. Nikolov, V. Panov, G., Psychological assesment of anger and aggression., Trakia Journal of Science, Vol.3, Number 4, 61-64, 2005

8. Servaes, P. Gielissen, MF. Verhagen, S. Bleijenberg, G., The course of severe fatigue in disease-free breast cancer patients: a longitudinal study. Psychooncology, 16:787-95, 2007

9. de Vries, U. Reif, K. Peterman, F., Top of Form Bottom of Form: Tumorbedingte Fatigue und ihre psychosozialen Belastungen November, 85-92, 2011

10.Minton, O. Stone, P., How common is fatigue in disease-free breast cancer survivors? A systematic review of the literature. Br Cancer Res Treat., 9831-1. 2007

11.Petkova, M. Nikolov, V. Hristakieva, E. Depressive symptoms in schizophrenia among older adults, Trakia Journal of Sciences, 5: 15-20, 2007

12.Mock, V. Atkinson, A. Barsevick, A. et al., Panel. NCCN practice guidelines for cancer-related fatigue (Version 1.2003). $J$ Natl Comp Cancer Network, 1308 -33, 2003

13.Bender, C. Kramer, P. Miaskowski, C. New direction in the management of cancerrelated cognitive impairment fatigue and pain. Pittsburgh: Oncology Nursing Society, 2002.

14.Patrick, DL. Ferketich, SL. Frame, PS. et al. Symptom management in cancer: pain, depression, and fatigue. National Institutes of Health state-of-the-science conference statement, July, 15-17, 2002. 
15.Glaus, A., Assessment of fatigue in cancer and non-cancer patients and in healthy individuals. Support Care Cancer, 1:305315, 1993

16.Zigmond, AS. Snaith, PR., The Hospital Anxiety and Depression Scale. Acta Psychiatr Scand, 67: 361-370, 1983

17.Mendoza, TR. Wang, XS. Cleeland, CS et al., The rapid assessment of fatigue severity in cancer patients: use of the Brief Fatigue Inventory. Cancer, 85(5): 1186-1196, 1999

18. Aaronson, N. Ahmedzai, S. Bergman, B. Bullinger, M. Cull, A. Duez, N. Filiberti, A. Flechtner, H. Fleishman, S. de Haes, J. Kaasa, S. Klee, M. Osoba, D. Razavi, D. Rofe, P. Schraub, S. Sneeuw, K. Sullivan, M., The European Organization for Research and Treatment of Cancer QLQC30: A quality-of-life instrument for use in international clinical trials in oncology. Journal of the National Cancer Institute, 85:365-376, 1993

19.Knobel, H. Loge, JH. Brenne, E. et al., The validity of EORTC QLQ-C30 fatigue scale in advanced cancer patients and cancer survivors. Palliat Med, 17(8):664-672. 2003

20.Storey, DJ. Waters, R. Hibberd, CJ. et al,. Clinically relevant fatigue in cancer outpatients: the Edinburgh Cancer Centre symptom study. Ann Oncol, 18(11):18611869, 2007

21.Smets, EM. Garssen, B. Bonke, B. et al., The Multidimensional Fatigue Inventory (MFI) psychometric qualities of an instrument to assess fatigue. $J$ Psychosom Res, 39(3):315-325, 1995

22.Smets, EMA. Visser, MR. Willems-Groot, AF. et al., Fatigue and radiotherapy: (A) Experience in patients undergoing treatment. Br J Cancer,78:899-906, 1998

23.Holzner, B. Kemmler. Greil, R. et al., The impact of hemoglobin levels on fatigue and quality of life in cancer patients. Ann Oncol, 13:965-973, 2002

24.Bartsch, HH. Weis, J. Moser, MT., Cancerrelated fatigue in patients attending oncological rehabilitation programs: Prevalence, patterns and predictors. Onkologie, 26:51-57, 2003

25. Howell, SJ. Radford, JA. Smets, EMA. et al., Fatigue, sexual function and mood following treatment for haematological malignancy: The impact of mild Leydig cell dysfunction. $\mathrm{Br} J$ Cancer, 82:789-793, 2000

26.Chalder, T. Berelowitz, G. Pawlikowska, T. et al., Development of a fatigue scale. $J$ Psychosom Res., 37(2):147-153, 1993
27.Loge, JH. Ekeberg, O. Kaasa, S. et al., Fatigue in the general Norwegian population: normative data and associations. J Psychosom Res., 65:53-65, 1998

28.Loge, JH. Abrahamsen, AF. Ekeberg, O. et al., Hodgkin's disease survivors more fatigued than the general population. J Clin Oncol.,17(1): 253-261, 1999

29.Hann, DM. Jacobsen, PB. Azzarello, LM. et al., Measurement of fatigue in cancer patients: development and validation of the Fatigue Symptom Inventory. Qual Life Res., 7(4):301-310, 1998

30.Hann, DM. Garovoy, N. Finkelstein, B. et al., Fatigue and quality of life in breast cancer patients undergoing autologous stem cell transplantation: A longitudinal comparative study. $J$ Pain Symptom Manage, 17:311-319, 1999

31.Jacobsen, PB. Garland, LL. Booth-Jones, M. et al., Relationship of hemoglobin levels to fatigue and cognitive functioning among cancer patients receiving chemotherapy. $J$ Pain Symptom Manage, 28:7-18, 2004

32.Broeckel, JA. Jacobsen, PB. Horton, J. et al., Characteristics and correlates of fatigue after adjuvant chemotherapy for breast cancer. J Clin Oncol., 16:1689-1696, 1998

33.Piper, BF. Dibble, SL. Dodd, MJ. et al., The revised Piper Fatigue Scale: Psychometric evaluation in women with breast cancer. Oncol Nurs Forum, 25:677684, 1998

34.Andrykowski, MA. Curran, SL. Lightner, R., Off-treatment fatigue in breast cancer survivors: A controlled comparison. $J$ Behav. Med., 21:1-18, 1998

35.Woo, B. Dibble, SL. Piper, BF. et al., Differences in fatigue by treatment methods in women with breast cancer. Oncol Nurs Forum, 25:915-920, 1998

36. Yasin, M. Areej, Al-Hamad., Anxiety and Depression as key determinants of cancer related faigue among patients receiving chemotherapy. European Scientific Journal, November edition vol.11, No.33, 2015

37.Schwartz, AL. The Schwartz Cancer Fatigue Scale: testing reliability and validity. Oncol Nurs Forum, 25:711-717, 1998

38.Schwartz, AL., Fatigue mediates the effects of exercise on quality of life. Qual Life Res., 8:529-538, 1999

39.Schwartz, AL., Daily fatigue patterns and effect of exercise in women with breast cancer. Cancer Pract., 8: 16-24, 2000

40.Glaus, A. Fatigue in patients with cancer. Analysis and assessment. Recent Results Cancer Res., 145:I-XI, 1-172, 1998 
41.Okuyama, T. Akechi, T. Kugaya, A. et al. Development and validation of the Cancer Fatigue Scale: a brief, three-dimensional, self-rating scale for assessment of fatigue in cancer patients. J Pain Symptom Manage, 19:5 14, 2000

42.Petkova, M. Nikolov, V. Galabova, M., Petrova Psychological assessment of cancer patients with chronic pain. Procedia Social and Behavioural Sciences, 5: 421425, 2010

43. Nikolov, V. Petkova, M. Pain and Social Activity in Colorectal Cancer PATIENTS. European Health Psychologist, 16: S,857, 2014 